\title{
The Role of Dominant Mutations in the Population Expansion
}

\author{
Stanisław Cebrat ${ }^{1}$ and Andrzej Pȩkalski ${ }^{2}$ \\ 1 Department of Genomics, Institute of Genetics and Microbiology, University of \\ Wrocław, \\ ul. Przybyszewskiego 63/77, PL-54148 Wrocław, Poland \\ cebrat@microb.uni.wroc.pl \\ 2 Institute of Theoretical Physics, University of Wrocław, \\ pl Maxa Borna 9, 50-204 Wrocław, Poland \\ apekal@ift.uni.wroc.pl
}

\begin{abstract}
We have studied the dynamics of expansion of diploid, sexually reproducing populations onto new territories demanding different genetic adaptations. Populations succeeded in the new habitats if the mutations of alleles responsible for adaptation to the new conditions were recessive. If behind the hostile territory another friendly habitat exists, several different scenarios are possible, including one in which the middle, most hostile, habitat remains empty, separating populated two, more friendly, ones.
\end{abstract}

\section{Introduction}

Sexual reproduction is one of the most interesting phenomena in Nature. Thus, it is obvious that even physicists are interested in this strategy, though without spectacular successes (negative birth rate is observed in the physics departments all over the world). Some of them even succeeded in proving that the strategy of sexual reproduction is a big misunderstanding of Nature and asexual populations would have much higher reproduction potential than sexual ones [1]. Such conclusions can be reached as the result of an evolution simulation if dominance of defective alleles is assumed. In fact, in Nature genomes posses a lot of redundant information and one of the simplest ways to get redundancy at all genetic loci is to be diploid. Organisms can profit from redundancy if the mutated information (defective gene) is recessive which means that its defect can be complemented by the proper gene - the allele of the defective one in the diploid genome (wild copy of a gene). If the mutated gene dominates and expresses defective phenotype, then increasing the number of copies of such genes is rather a loosing strategy. That is why the number of loci in diploid genomes where mutations lead to dominant deleterious functions is low. In many instances, like mutations in oncogenes, it may be even connected with a special Nature's policy - elimination of old dispensable organisms (professors). Sexual strategy of reproduction usually needs two well balanced sets of information because these sets should be separated into two equivalent pools of genes in gametes which, 
joining in pairs, produce new individuals. It seems reasonable that it would still be better to increase the redundancy by forming triploids or tetraploids. But even neglecting the costs of replication of the redundant information it could be a loosing strategy if there were too many loci with potentially dominant mutations in the genomes 2. Nevertheless, dominance is a rather complicated problem in biology. It is possible that a mutation in the same pair of alleles can be recessive in one condition or for one phenotype while dominant in another one. Let's imagine a pair of alleles producing an enzyme which splits a toxic compound. If one allele of the pair is mutated the toxic compound can still be eliminated from the organism - the mutation is recessive. What would happen if the mutation in the gene produced an enzyme which would transform the other compound into a cancerogen - a product promoting cancerogenesis ? If the organism is exposed to this pre-cancerogenic compound such a mutation behaves as dominant. Thus, the status of mutations could depend on some environmental conditions. Immunology supports some other examples of complicated relations between different alleles of the same locus and environment [3]. The distribution of the main blood group antigens A,B,O in the human population is very uneven on Earth. If an individual has antigen A, it cannot produce antibodies anti-A because it would develop an auto-immune disease. If in the environment there is a pathogen with A-like antigens, organisms cannot develop the immune response against this antigen. Some pathogens are known which can use such a mimicry to find a defenseless host. Some pathogens can be so promiscuous that they use mimicry to invade the host and finally they may induce cancerogenesis [4. In our simulations we tried to show that redefinition of loci from recessive to dominant is much more dangerous for the survival of the whole population than redefinition of the value of alleles in the locus from positive to negative, keeping the dominance unchanged.

\section{Model}

We consider a population composed initially of $N(0)$ individuals located at the leftmost part of the square lattice, of dimensions $150 \times 50$ lattice sites, forming our system. Each individual, apart from its location on the lattice is characterized by its genotype $g$ and age age. The genotype is a double string of length $L=$ 32 of zeros and ones. From the genotype the individual's phenotype, $f$, also of length $L$, is constructed as a product of the values at corresponding loci. The rules of taking the product depend whether a zero is declared at the dominant allele, or not. The population is under the influence of the external "field ", which could be the climate, represented as the optimal phenotype, $F$, of length $L$ with components equal either zero or one. The optimal phenotype changes along the $X$-axis. The system is divided into three regions, labelled later $I, I I, I I I$, with the corresponding optima $F_{I}, F_{I I}, F_{I I I}$. At the leftmost region $(I)$ the optimum is just a string of zeros. In the second region a certain number, Lch, of 0's is switched to 1's. In the third region either there are twice as many changes as in the region II, or the "climate" is the same is in the region I. 
At the beginning the population has random values of their genotypes and locations on the lattice. The algorithm goes as follows

1. an individual $j$ is picked at random,

2. its adaptation to the external conditions, i.e. the agreement between its phenotype and the optimal one is calculated from the formula

$$
a d_{j}=\frac{1}{32}\left(1-\sum_{\alpha=1}^{32} \operatorname{XOR}\left(f_{j}^{\alpha}, F^{\alpha}\right)\right),
$$

where the $F$ is taken according to the position of the individual in the system (in the I, II or III region),

3. its survival probability is determined

$$
p_{j}=\exp \left(- \text { sage }_{j} / a d_{j}\right),
$$

where $s$ is the selection pressure. A random number $r \in(0,1)$ is drawn. If $r>p_{j}$ the individual is removed from the system. Otherwise

4. a search is made in the nearest neighborhood for an empty place to move the individual. If successful and the age of the individuals is greater then one (to eliminate coupling of offspring right after birth)

5. another search is made in the nearest neighborhood of the new position for a partner. Once found

6. the pair produce at most 4 offspring, each of which receives its genotype independently via recombination and crossing over of the parents' genotypes. Each loci of the offspring could be mutated with a probability $p m$, which is a parameter of the model. Independent search is made in the Moore neighborhood ( 8 sites nearest to the first parent) to put an offspring. If a search was not successful, the progeny is not born. This is the only place where a kind of Verhulst factor regulates the density of the population.

When, at a time $t$, as many individuals were picked as first partners, as was the total number of individuals at that time, i.e. $N(t)$, one Monte Carlo step has been made and the age of all individuals is incremented by one. In the following, time is measured always in Monte Carlo steps (MCS). This kind of models has been used before by us in different contexts [5].

\section{Results and Discussion}

Populations simulated under the chosen selection pressure and mutational pressure parameters are stable and survive the prolonged simulations. In these populations, mutations in all alleles of all loci were recessive. If we re-declare the values of some loci which means that the new environment requires different alleles in these loci, the populations eventually adapt to the new conditions and approximate to the concentration usual for such conditions (Fig. 1).

The situation is much more dangerous for the simulated populations if the redefinition of the loci values is connected with the declared dominance of the 

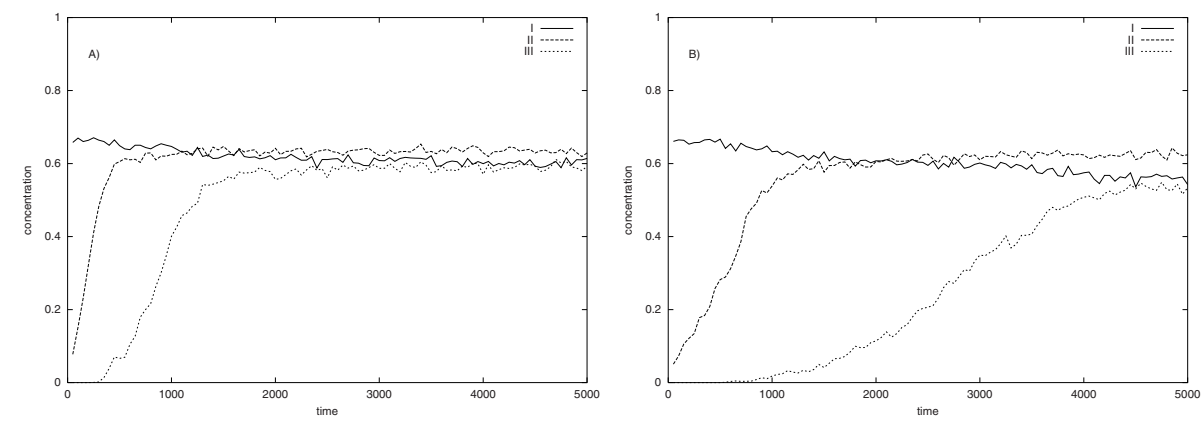

Fig. 1. Expansion of populations on the new territories without changes in dominance conditions. A) II region demands different values in 4 loci, III region demands different values in 8 loci. B) II region demands different values in 7 loci, III region demands different values in 14 loci. Mutations at all loci stay recessive. Average over 10 runs. Three lines correspond to the three regions
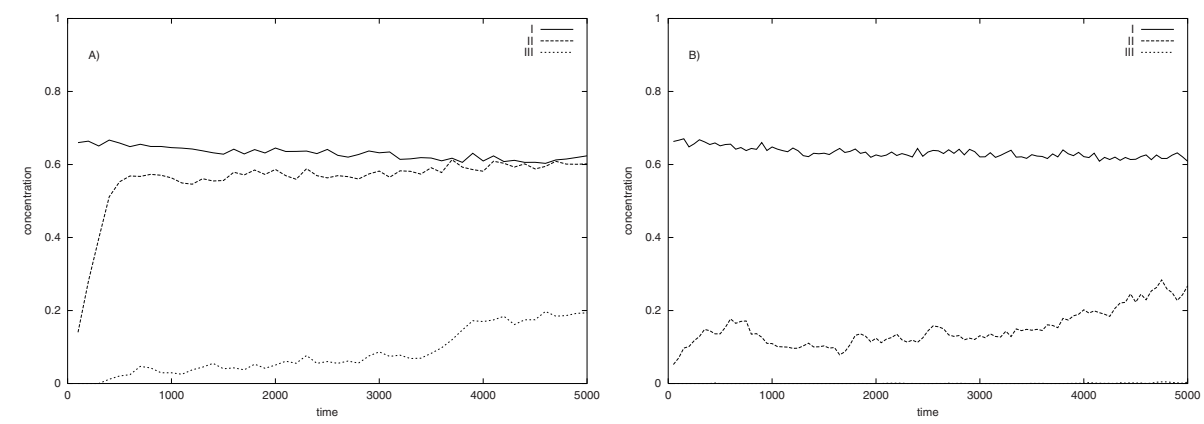

Fig. 2. Expansion of populations on the new territories with changes in dominance conditions. A) II region demands different values in 4 loci, III region demands different values in 8 loci. B) II region demands different values in 7 loci, III region demands different values in 14 loci. Mutations at all loci are dominant. Average over 10 runs. Three lines correspond to the three regions

mutated genes. The chance for survival of the population depends strongly on the number of declared dominant loci (Fig. 2).

In our simulations we have assumed that the territory where the population evolves is divided into three sectors. Initially the population occupies one sector (left) and it is adapted to the environmental conditions in it, with all mutations recessive.

Conditions in the middle sector require different values of some genes and in one case mutations in these loci are recessive, while in the second case these mutations are dominant. In the first case the population invades the second sector, adapts to the new conditions and eventually reaches the third sector which still demands the new adaptations. In the second case the population cannot permanently occupy the middle sector. Some trials are observed of settling the 


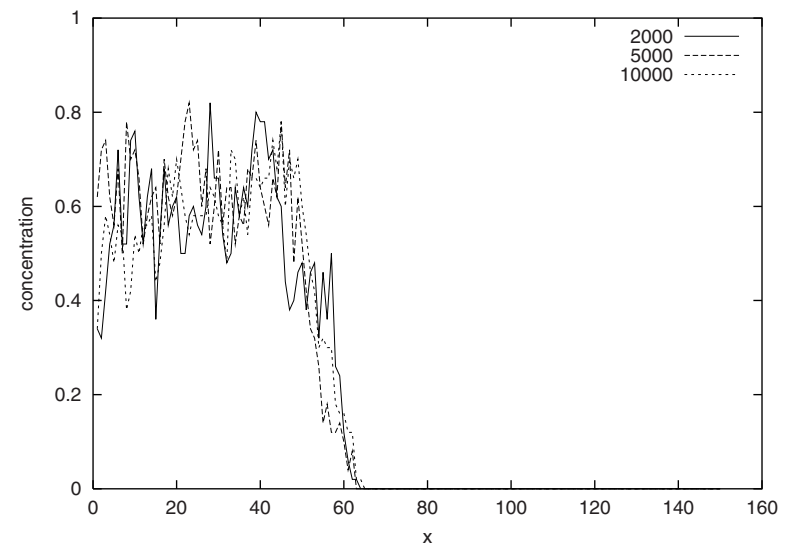

Fig. 3. Spatial distribution of a population at three time steps - 2000, 5000 and 10000 MCS. Zero is the dominant allele and the number of changes in the optimum is 7 in the II and 14 in the III region, respectively

territory close to the border, but concentration of the population drops very quickly with the distance to the friendly maternal territory (Fig. 3).

To test the possibility of crossing the hostile territory we have declared that the third sector (the right one) is as friendly as the first one and it requires the same phenotypes as the first one, but the initial population was settled on the first sector only (Fig. 4). It is obvious that like in the above simulations, if there were no dominant deleterious mutations, populations expanded onto all three sectors with approximately the same concentrations in all of them. But if the mutations in the re-declared loci were dominant, the populations were not able to cross the hostile territory. Only in some rare instances they succeeded in penetrating this sector. Having reached the new territory with the same environmental conditions as the initial one, the population occupied it very fast. The interesting observation is that the middle hostile territory is not desolate any more, though the population is less concentrated in it.

It should be noted that the problem of dominance has been recently raised by the modern eugenics techniques. In the so called "risk cases" the fertilization in vitro and embryo transfer followed by preimplantation genetic diagnosis is recommended. It is obvious that heterozygotic embryos are eliminated even in case of recessive mutations. Such a procedure mimics the dominance of mutations. For more discussion of eugenics problem see also [6]. Further studies should answer the question whether the hostile territory in the reach of populations ameliorates the genetic status of the whole population.

\section{Conclusion}

It has been shown that it is important to consider the dominance of alleles when studying the sexual strategy of reproduction. Populations can expand to new 

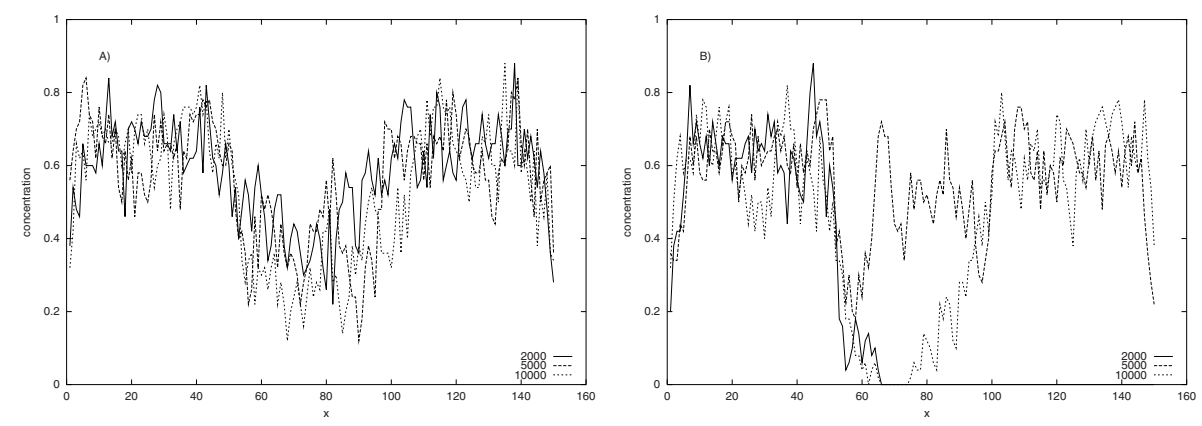

Fig. 4. As in Figure 3, except that now the optimum is the same (more friendly) in the regions I and III. Presented are two populations, starting with similar initial conditions

territories and adapt to new conditions relatively fast if it is not connected with the appearing of new dominant loci. The process of penetration of the hostile territory probably selects very well adapted individuals.

Acknowledgements. Authors thank J. Mleczko and J. Kołodyński for discussions. The work is a part of the program of the COST Action P10.

\section{References}

1. Redfield RJ, Nature, 369 (1994) 145.

2. Sousa AO, Moss de Oliveira S, Sa Martins, Evolutionary advantage of diploidal over polyploidal sexual reproduction. Phys.Rev. E 67 (2003) 032903

3. Oldstone MB, Molecular mimmicry and immune mediated diseases. FASEB J. 12 (1998) 1255.

4. Logan RP, Helicobacter pylori and gastric cancer. Lancet 344 (1994) 1078.

5. A. Pȩkalski and K. Sznajd-Weron, Population dynamics with and without selection, Phys.Rev. E 63, 031903, 2001

6. S. Cebrat and A. Pȩkalski, Model of population evolution with and without eugenics, Eur.Phys.J. B 11, 687, 1999 